\title{
Tactical UGV Navigation and Logistics Planning
}

\author{
Talib S. Hussain \\ BBN Technologies \\ 10 Moulton St. \\ Daniel Cerys \\ BBN Technologies \\ 10 Moulton St. \\ David Montana \\ BBN Technologies \\ 10 Moulton St. \\ Cambridge, MA 02138
617-873-6861 \\ 617-873-4534 \\ thussain@bbn.com \\ Gordon Vidaver \\ BBN Technologies \\ 10 Moulton St.
Jeffrey E. Berliner
BBN Technologies
10 Moulton St. \\ 617-873-2719 \\ 617-873-3558 \\ 617-873-3835 \\ cerys@bbn.com dmontana@bbn.com gvidaver@bbn.com berliner@bbn.com
}

\begin{abstract}
The Army's push towards developing highly flexible military teams that combine manned and unmanned units requires significant advances in the intelligence of the unmanned units and in the tools used to provide logistical support. BBN Technologies has recently completed a simulation-based project for the Army Research Lab in which we applied an evolutionary computation approach for determining the tactical responses of an unmanned ground vehicle, moderated by explicit rules of engagement. BBN is also currently developing a logistic analysis prototype that uses agent-based technology and evolutionary computation to enable rapid logistics planning and replanning for supporting an Army organization encountering a diversity of planned and unplanned situations.
\end{abstract}

\section{Categories and Subject Descriptors}

I.2.9 [Artificial Intelligence]: Robotics - Autonomous vehicles; I.2.8 [Artificial Intelligence]: Problem Solving, Control Methods and Search - Plan execution, formation, and generation; J.7 [Computers in Other Systems]: Military.

\section{General Terms}

Algorithms, Design.

\section{Keywords}

Tactical behaviors, unmanned ground vehicles, logistics planning

\section{INTRODUCTION}

In [1], we presented our Advocates and Critics for Tactical Behaviors (ACTB) technique for unmanned ground vehicle (UGV) navigation and our efforts in developing a support tool for supporting a Future Combat Systems (FCS) Unit of Action (UA). We have extended both avenues of research significantly in the past year. We conducted a project for the Army Research Lab (ARL) in which we applied our ACTB technique to an ARL UGV simulator in which we faced mobile threats. Our technique was extended in several ways to enable real-time planning and response, as well as to allow explicit bias of the evolutionary search under different tactical situations. We are currently

() 2005 Association for Computing Machinery. ACM acknowledges that this contribution was authored or co-authored by a contractor or affiliate of the [U.S.] Government. As such, the Government retains a nonexclusive, royalty-free right to publish or reproduce this article, or to allow others to do so, for Government purposes only.

GECCO'05, June 25-29, 2005, Washington, D.C., USA.

Copyright 2005 ACM 1-59593-097-3 ...\$5.00. conducting a project in which we are developing a tool that extends our previous work on FCS supportability by making it more robust, providing the support for a wider variety of items and support relationships, and performing two-tier scheduling using evolutionary computation. We briefly recap each technique, present the key changes made, and discuss both the motivations for those changes and the benefits that have resulted.

\section{TACTICAL UGV NAVIGATION}

The ACTB approach, as presented in $[1,2]$, was based upon the use of an evolutionary search algorithm in which domain-specific operators were applied to a variable-length representation of the paths of one or more vehicles. The representation was a plan that identified the sequence of future waypoints to be visited by each vehicle. The domain-specific operators were termed advocates, and made modifications to the plan that reflected certain tactical behaviors, such as trying to circle around a known enemy area. The fitness function was the weighted sum of the results of a number of critics. Each critic evaluated the plan against a specific tactical goal, such as how quickly the plan would enable the vehicles to achieve the mission objectives. In [1,2], our system only dealt with unmoving enemies and there was no imperative for real-time behavior

In the project for ARL, we were presented with a new simulation environment and new tactical objectives. The simulator was developed by General Dynamics Robotics Systems (GDRS), and defined a simple world (see Figure 1) 640 meters by 480 meters in size consisting of obstacles (in black) and clear areas (in white). Within the world, there were three flag locations, all randomly located within 100 meters of each other. Our goal was to control a single simulated UGV and to compete against one or two other simulated UGVs. The competition consisted of maximizing the number of flags visited, and to either survive for a fixed period of time or kill the opponent(s) within that time. The simulated UGV had a simulated gun turret and a simulated camera, and could be controlled via five basic commands. The vehicle could move to a given geographical location, stop movement, look in a given direction by rotating the camera, aim the gun in a given direction, and fire

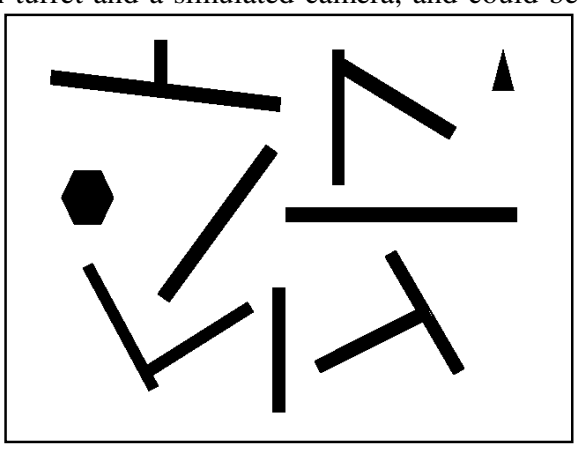

Figure 1: Sample simulated terrain 
the gun. The simulator performed in real time, typically with half a second delay between issuance of a command and execution of that command. Information (e.g., from looks) was also provided in a similar time frame.

We adopted a genetic representation that specified a sequence of waypoints to visit and (zero or more) look actions to perform at each of those waypoints. Gun control and firing commands were determined by an independent, opportunistic control system (i.e., automatically target and fire at nearby enemies). As in [1,2], advocates and critics for specific tactical behaviors were created. For example, we developed advocates that would encourage the UGV to stay close to walls, or look around when approaching a corner; we developed critics that would evaluate how much risk of being seen a plan put the vehicle in, or how aware of its surroundings a vehicle would be based on its planned looks. We also biased the advocates to operate on portions of the plan that were sooner in time, and portions of the plan that had been identified as very poor by one or more critics. These changes to the advocates and critics led to rapid planning of realistic tactical behaviors under many circumstances. However, we discovered that the system often performed poor real-time planning when the tactical situation changed significantly. For example, if an enemy was discovered, our system spent a lot of time thrashing as it tried to replan.

To address this, we adapted the ACTB architecture to incorporate high-level strategic "rules of engagement" that influenced the manner in which planning was performed. In particular, the strategy rules determined the current attitude of the system. An attitude was a consistent set of advocates and critics, each with certain probabilities of selection and weights, respectively. When

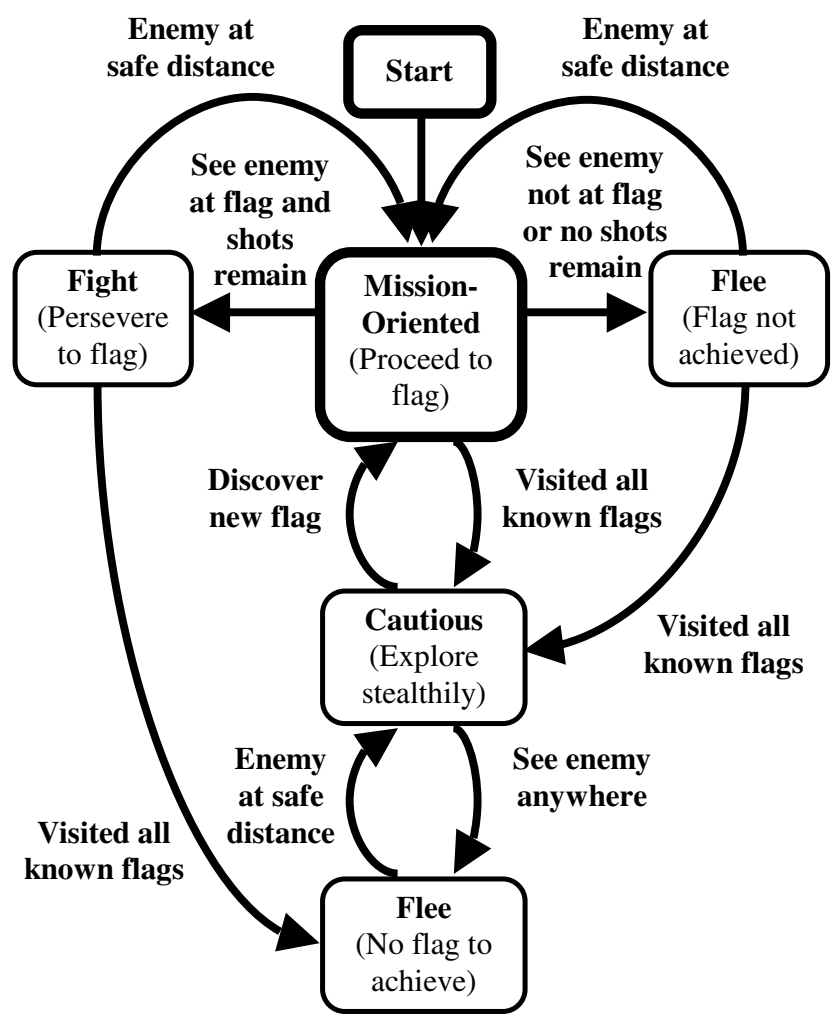

Figure 2: Rules of engagement for attitude transitions the system's attitude changed, the advocates and critics used by the planning process were immediately changed to reflect those specified in the new attitude. Thus, instead of trying to solve one difficult optimization problem, the revised ACTB approach used attitudes to solve several simpler ones to improve the quality and timeliness of its response. This reflects, for example, the fact that during a mission it may be appropriate under different conditions for a UGV to become more cautious (e.g., it doesn't know where the enemy is so try to stay out of sight) or more aggressive (e.g., the enemy is in the way of reaching a flag, so close and attack). Figure 2 illustrates the rules of engagement used to determine when to transition from one attitude to another.

Through the use of advocates, critics and attitudes, ACTB naturally admits the diversity of goals and constraints that are important for the tactical behavior navigation problem, but in a manner that makes those aspects easy to understand. In September 2004, our system was tested head-to-head, in over one hundred trials, against two other state-of-the-art tactical behavior systems (developed by two competing organizations) and performed very well.

\section{LOGISTICS ANALYSIS}

In [1], we presented a scalable, agent-based modeling system using Cougaar agent technology [3] to represent the logistics activities of the Army's Future Combat Systems' (FCS) Unit of Action (UA) and to generate refueling requests, and using BBN's Vishnu [4] genetic algorithm software to assign refueling requests to the refueling trucks. In particular, the system examined the problem of distributing fuel to the combat vehicles within the UA over a 72 hour period, where all fuel for resupply was stored on the refueling trucks that moved within the UA (i.e., no fixed supply depots). The GA optimized against multiple goals, including minimizing the travel performed by the refueling trucks, minimizing the number of refueling trucks needed and maximizing the number of requests that are satisfied (on time and with required quantities).

We have extended our work to incorporate scheduling the supply of both ammunition and fuel. The ammunition and fuel supply problems are solved simultaneously and independently. Ammunition scheduling is like fuel scheduling except that there are multiple types of ammunition versus just one type of fuel, and each request for a specific type of ammunition must be drawn from a container with that particular type in it. Further, while fuel scheduling has a flexible delivery amount based on the time delivered (e.g., the scheduler can satisfy a request by delivering less fuel earlier, or more fuel later, rather than the exact amount at the exact time requested), ammunition scheduling is different there is no time dependency on the amount delivered. For example, for a customer that uses 1 gallon/hour, if the request is for 100 gallons at 12:00, the scheduler may deliver 97 gallons at 09:00. In contrast, the only time a partial ammunition response will be given (e.g., asking for 10 rounds but getting 7) is when there is an insufficient amount available on the supply trucks.

This system has been extended to model evolving military logistics doctrine. In the FCS UA, the primary combat units now have their own organic support units with whom they operate in close coordination. These support units resupply the combat vehicles during specified time periods of low combat intensity, and are themselves resupplied from the dynamically located 


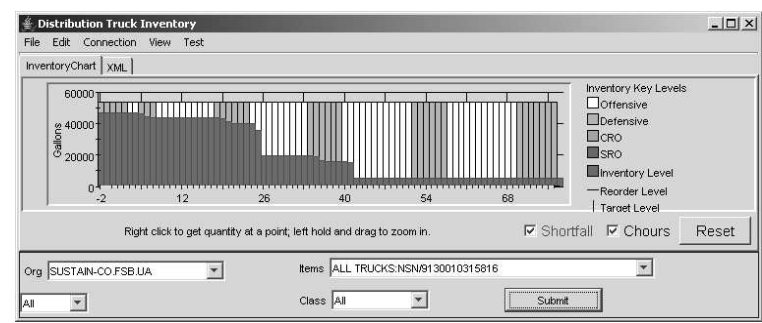

(a) Dynamic inventory optimization at FSB.

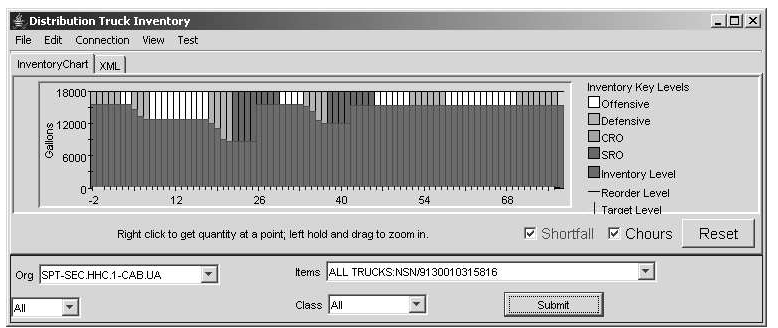

(b) Dynamic inventory optimization at Support Unit.

Figure 3. Two-tier scheduling with dynamic inventory optimization

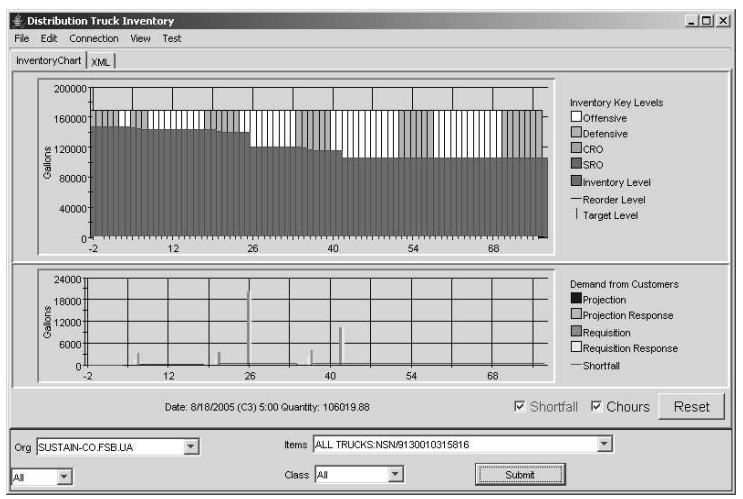

(a) 46 fuel trucks at FSB

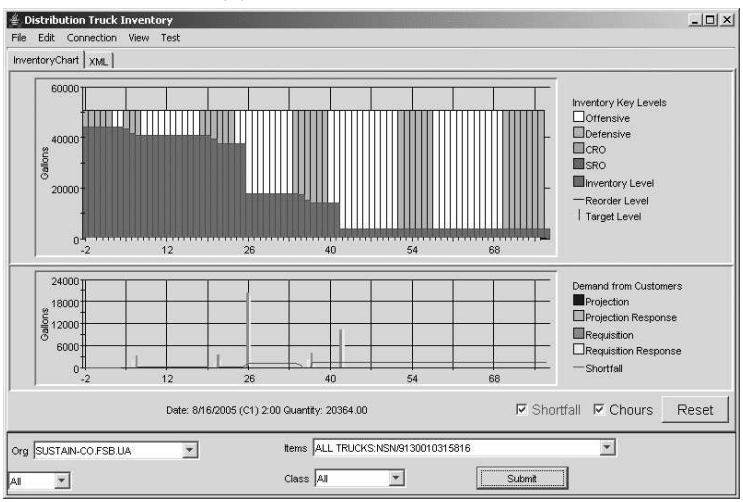

(b) 11 fuel trucks at FSB

Figure 4: Optimization of number of fuel trucks at FSB required for support. (a) 46 fuel trucks case showing over 100,000 excess gallons of fuel after 72 hour support period, (b) 11 fuel trucks case showing 3200 excess gallons.
Forward Supply Battalion (FSB) at different times. To model this doctrinal change, the extended system performs two-tier supply and dynamic inventory optimization.

The tool schedules the delivery of supplies to the end customer (e.g., FCS combat unit) for a certain period of time, and then schedules the re-supply of the supply vehicles themselves from the FSB to replace the amount expected to be delivered. This two-tier supply is performed using dependent evolutionary searches (using Vishnu), and repeats in a cycle as new supply demands are received. Between cycles, the number of supply trucks that are in use may be adapted dynamically based on expected load. Figure 3 shows the process of the two-tier supply and the combined effect of re-supply and dynamic inventory optimization. The support unit receives fuel from the FSB (bars increase) and optimally distributes it to its customers (bars decrease). Figure 4 illustrates the effect of a reduction in the number of fuel trucks assigned to the FSB from 46 to 11, in an effort to minimize the logistics footprint, while maintaining an adequate supply over 72 hours. The bars indicate the total fuel remaining in all the trucks at the FSB in the two cases as time passes.

\section{CONCLUSIONS}

Real-time tactical behavior planning using evolutionary computation was achieved through the incorporation of multiple independent search biases (or attitudes) and high-level rules for changing the current bias under specific tactical situations. Effective planning and replanning of multiple types of logistic support items with two tiers of supply vehicles was achieved using evolutionary computation.

\section{ACKNOWLEDGMENTS}

The ACTB work was conducted for the Army under subcontract to General Dynamics Robotics Systems (GDRS), contract \#DAAD19-01-C-0066.

\section{REFERENCES}

[1] Hussain, T., Montana, D., Brinn, M. and Cerys, D. Genetic algorithms for UGV navigation, sniper fire localization and Unit of Action fuel distribution. Workshop on Military and Security Applications of Evolutionary Computation (MSAEC-2004) at GECCO-2004. S. Upton and D. Goldberg (Organizers), CD_ROM Proceedings, 2004.

[2] Hussain, T., Montana, D. and Vidaver, G. Evolution-based deliberative planning for cooperating unmanned ground vehicles in a dynamic environment. Genetic and Evolutionary Computation - GECCO 2004, Part II. Lecture Notes in Computer Science 3103. K. Deb et al (Eds.), Springer-Verlag, Berlin, 2004, 1017-1029.

[3] “Cougaar Open Source Web Site,” http://www.cougaar.org/.

[4] Montana, D. Optimized scheduling for the masses. GECCO2001 Workshop: The Next Ten Years of Scheduling Research, 2001. 